Research Article

\title{
The Epoxidized Vietnam Rubber Seed Oil as a Secondary Plasticizer/Thermal Stabilizer in PVC Processing
}

\author{
Nguyen Thi Thuy (D), Vu Minh Duc, and Nguyen Thanh Liem \\ Hanoi University of Science and Technology, Hanoi 10000, Vietnam \\ Correspondence should be addressed to Nguyen Thi Thuy; thuy.nguyenthi1@hust.edu.vn \\ Received 19 February 2021; Revised 4 March 2021; Accepted 27 March 2021; Published 12 April 2021 \\ Academic Editor: Mehdi Salami-Kalajahi
}

Copyright (c) 2021 Nguyen Thi Thuy et al. This is an open access article distributed under the Creative Commons Attribution License, which permits unrestricted use, distribution, and reproduction in any medium, provided the original work is properly cited.

\begin{abstract}
The epoxidized rubber seed oil (EeRSO) was a mixture of epoxidized triglyceride, and epoxidized methyl ester of free fatty acids was used as a secondary plasticizer for PVC. An increase in tensile properties was observed by substituting the 10 phr DOP plasticizer with the EeRSO in PVC formulation. A leaching test was performed in five media to evaluate the plasticizing effect. The sample weight increased slightly after soaking in water and $30 \mathrm{wt} . \%$ acetic solution, decreased slightly in $10 \mathrm{wt} . \% \mathrm{KOH}$ solution, and reduced sharply and strongly in sunflower oil and n-hexane. The $10 \mathrm{phr}$ EeRSO in PVC formulation has presented an improvement in migration, volatilization characteristics, and thermal property of PVC. After 72 hours of soaking in n-hexane, the shore A hardness of the EeRSO plasticized PVC sample increased by $14.5 \%$ while the PVC sample without EeRSO was blistered and its shore A hardness could not be measured. This was the clearest evidence for the positive effect of EeRSO as a secondary plasticizer. The morphology of the fractured surface of the samples after immersing in n-hexane was studied by using scanning electron microscopy. Thermogravimetric analysis showed the role of EeRSO in significant improvement in thermal stability. In general, EeRSO not only acts as a primary plasticizer to improve the migration, extraction, and volatilization characteristics but also contributes to the thermal stability of PVC.
\end{abstract}

\section{Introduction}

Poly(vinyl chloride) (PVC) is one of the most widely consumed thermoplastics worldwide for numerous applications such as wire and cable insulation, flooring, wall cover, packaging materials, children toys, infant supply, and medical supplies [1-4]. The presence of a C-Cl dipole bond in each repeating unit restricts chain mobility so PVC has hard, brittle characteristics $[2,4-6]$ and low thermal stability due to loss of $\mathrm{HCl}$ [7-11]. In order to improve PVC characteristics, additives should be added. The plasticizers and thermal stabilizers were used usually in PVC processing. The plasticizers can be classified as internal and external [12] or can also be either defined as primary or secondary plasticizers [13]. In which, phthalate ester-petroleum-based products are the most commonly used plasticizer family $[5,6]$. However, most plasticizers are well-known to be toxic $[6,14]$ and not chemically bonded with the PVC polymer chains, so they will leach from products into surrounding media $[6,15]$. Therefore, they should be replaced with biobased or renewable, environmentally friendly resources $[5,6,14]$. The modified vegetable oil such as epoxidized vegetable oil is very suitable because it contains many epoxy groups and has renewable sources $[2,16,17]$. Moreover, it is not only known as a plasticizer, epoxidized vegetable oils but also contributes to improving the thermal stability of PVC [9]. There are many types of epoxidized vegetable oils such as soybean oil [18-20], linseed oil [21-24], and sunflower oil [25] that have been used as primary or secondary plasticizers and/or thermal stabilizers for PVC. Among them, rubber seed oil and its derivatives have not only been used for a long time $[7,8]$ but are also very popular because it is an inedible oil [26]. The research works related to rubber seed oil and PVC are also very diverse. The order of the stabilizing effect was found to be metal soaps of epoxidized rubber seed oil $>$ metal soaps of rubber seed oil $>$ epoxidized rubber seed oil $>$ rubber seed oil by Okieimen et al. when they studied the thermal degradation and dehydrochlorination of PVC in the presence of 
rubber seed oil $[7,8]$. In another publication of Balköse et al., the thermal stability studies, plasticizer efficiency, and permanence properties were used to assess characteristics of PVC plastigels using epoxidized rubber seed oil with $4.5 \%$ oxirane content [11]. The dynamic mechanical analysis, hot air aging tests, and color stability tests in the study of Joseph et al. indicated that epoxidized rubber seed oil could be used as a less volatile secondary plasticizer and heat stabilizer for PVC in combination with dioctyl phthalate (DOP) [9]. Zinc soaps of rubber seed oil and/or epoxidized rubber seed oil were used as thermal stabilizers for PVC plastigels by Egbuchunam et al., and the effect of them was evaluated by the conductivity measurements and amount of $\mathrm{HCl}$ release [10]. Another rubber seed oil derivative as epoxidized dimeric acid methyl ester was also used as a secondary plasticizer for PVC, and its performance was evaluated through thermal properties, mechanical properties, and migration stabilities [27]. Thus, for the application of plasticizer or secondary heat stabilizer for PVC, scientists previously used rubber seed oil, epoxidized rubber seed oil, metal soaps of rubber seed oil, or epoxidized rubber seed oil $[7-9,11]$, epoxidized dimeric acid methyl ester [27] as separate or as a combination of metal soap rubber seed oil with epoxidized rubber seed oil [10].

In the present work, the author used an epoxidized rubber seed oil with the oxirane oxygen of 7.0-7.2\% as a secondary plasticizer for PVC. Here, the epoxidized rubber seed oil briefly referred to as EeRSO was a mixture of epoxidized triglyceride and epoxidized methyl ester of free fatty acids in high acid value rubber seed oil. The detail of EeRSO will be clearly explained in the section of materials. By partially replacing DOP in PVC formulations, a variety of characteristics of PVC such as tensile properties, migration, leaching and thermal property, and hardness were investigated. This is a huge difference of this article compared to the previous works.

\section{Materials and Methods}

2.1. Materials. The PVC TH-1000R with a $K$ value of $65.7-$ 67.7 was supplied by Taiyo Vinyl Corporation, Japan. The heat stabilizer was purchased from Huike, China. The processing aid Metablen 551J was provided by Mitsubishi Chemical, Japan. The DOP plasticizer (dioctyl phthalate $\mathrm{C}_{24} \mathrm{H}_{38} \mathrm{O}_{4}$ ), acid stearic 401, and PE wax SCG were of China, Indonesia, and Thai origin, respectively.

Rubber seeds were collected in Long Khanh, Dong Nai, Vietnam, after 3 months to dry were pressed in EC company in 364/1 Cong Hoa, Tan Binh, to obtain rubber seed oil (RSO) with iodine and acid value of $146.9 \mathrm{cgI}_{2} / \mathrm{g}$ and $39.7 \mathrm{mgKOH} / \mathrm{g}$, respectively. At first, RSO was modified with methanol to reduce the acid value $(1.12 \mathrm{mgKOH} / \mathrm{g})$ and then epoxidized by using a sodium tungstate dihydrate catalyst in the National Key Laboratory for Polymer \& Composite Materials, Hanoi University of Science and Technology. Chemical titration according to ASTM D1652 gives the oxirane oxygen of the epoxidation product as 7.0-7.2\%.

The fact is that the free fatty acid content in rubber seed oil is very high and they are usually removed by saponifica- tion with a $\mathrm{NaOH}$ solution to obtain a rubber seed oil with a low acid value before it is transformed into epoxy [26]. But in the National Key Laboratory for Polymer \& Composite Materials, these free fatty acids were utilized because the RSO was esterified with methanol (methanol/oil ratio of $7 / 1)$ under mild conditions $\left(60^{\circ} \mathrm{C}\right)$ to convert free fatty acids in the oil into methyl esters. All ingredients as triglycerides and methyl esters of free fatty acids in esterified RSO would be utilized to transform into epoxy form, so the product of epoxidation was also a mixture of epoxidized triglycerides and epoxidized methyl ester of free fatty acids. This product mixture was used as a secondary plasticizer for the PVC in the present work.

\subsection{Methods}

2.2.1. Sample Preparation. Firstly, PVC and additives (see Table 1) were premixed at $100^{\circ} \mathrm{C}$ in a mixing device at a mixing speed of $2400 \mathrm{rpm}$ for $10 \mathrm{~min}$ then cool quickly to $50^{\circ} \mathrm{C}$ and continue to mix for $5 \mathrm{~min}$ and then incubate at room temperature in 24 hours. Secondly, the Haake PoLylab System Rheomix was used to mix. The mixture was introduced gradually during the first minute. The mixing time and speed were kept constant with a duration of $5 \mathrm{~min}$ and $50 \mathrm{rpm}$ at temperature of $180^{\circ} \mathrm{C}$. Finally, the PVC-based sample was collected and flattened with a steel roller.

The PVC-based sample was heated at $200^{\circ} \mathrm{C}$ and pressed at $130 \mathrm{~kg} / \mathrm{cm}^{2}$ by using the Gotech model GT-70140-P30C to get thin films of $500 \mu \mathrm{m}$ thickness on average or cylindrical blocks of $8 \mathrm{~cm}$ diameter and $3 \mathrm{~mm}$ thickness. Once the films are made, they had to be shaped before testing. A manual punch press was used for this purpose. The tensile samples and cylindrical blocks are allowed to stabilize at $25^{\circ} \mathrm{C}$ and $\mathrm{RH}$ of $50 \%$ for 72 hours before they are subjected to analysis.

2.2.2. Testing Methods. Tensile strength of samples was determined according to ASTM D638 standard by using Lloyd, England. The thermal properties were studied by thermogravimetric analysis (TGA) on a (DTA/DSC/TGA) Labsys Evo S60/58988 (France). The morphologies of samples were analyzed by using a scanning electron microscope (SEM, JSM-6360/6360LV, Japan). The shore A hardness of samples was determined according to ASTM D2240 standard by using Teclock GS 709, Japan. The leaching of plasticizers from plasticized PVC sample was carried out based on ASTM D 1239-98 standard. The testing environment and method for the leaching test were the same in the previously published article [24]. Migration test and migration and volatilization test were carried out according to the previously published article [11, 24, 27].

\section{Results and Discussion}

3.1. Tensile Properties. Tensile properties depend not only on the nature of the plastic but also on the additives used in their composition. So, they are a good way to evaluate the efficiency of plasticizers. The presence of EeRSO affected strongly to tensile properties. The loading of EeRSO increased to the ratio of 50/10; the modulus of PVC materials reduced slightly, but it grew up when the loading of EeRSO 
TABLE 1: The compositions in PVC recipe.

\begin{tabular}{lcc}
\hline No. & Compositions & Value $(\mathrm{g})$ \\
\hline 1 & PVC & 100 \\
2 & DOP & Variable \\
3 & EeRSO & Variable \\
4 & Acid stearic & 0.2 \\
5 & PE wax & 0.5 \\
6 & Heat stabilizer & 2 \\
7 & Processing aid & 3 \\
\hline
\end{tabular}

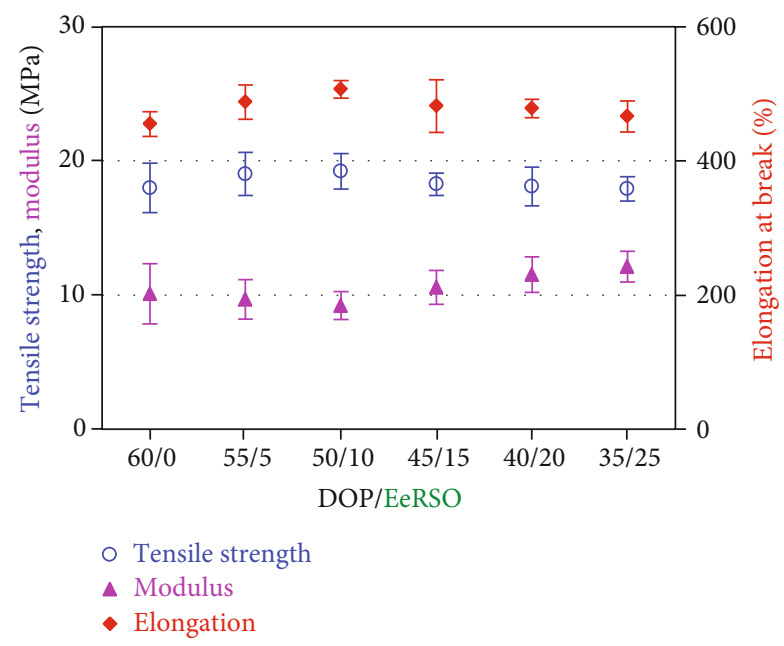

Figure 1: The effect of EeRSO content on the mechanical properties of PVC.

continued to raise EeRSO (see Figure 1). Unlike modulus, the tensile strength increased gradually from 17.97 to $19.22 \mathrm{MPa}$ when the loading of EeRSO increased to a ratio of 50/10. However, the loading of EeRSO after this point, the tensile strength of PVC materials reduced gradually, and it was nearly equivalent to PVC material using DOP only when the loading of EeRSO reached the ratio of 40/20 (see Figure 1).

The presence of EeRSO in PVC materials led to an increase in the elongation at break. The loading of EeRSO and DOP at the ratio of 50/10, the elongation at break of PVC material reached the largest and improved by $11.4 \%$ compared to the PVC material without EeRSO. The larger the EeRSO content, the elongation at break tended to decrease gradually (see Figure 1). Thus, the presence of EeRSO at a ratio of 50/10 had improved the tensile strength and elongation at break of PVC material while the modulus decreased by $8.7 \%$. Meaning, the presence of EeRSO has further improved PVC's flexibility. Undergoing heat in processing, PVC will be thermally decomposed by the dehydrochlorination. The generated hydrochloric acid contributes to promote the thermal decomposition of PVC. However, in the presence of epoxidized oil, the generated $\mathrm{HCl}$ will react with the epoxy group of the oil, resulting in minimizing thermal decomposition [10,25], so the tensile properties of PVC with the presence of epoxidized oil are slightly improved.
3.2. Leaching Property. Two samples with (50/10) and without $(60 / 0)$ EeRSO were used to the investigation of leaching characteristic in media as water, acetic acid and potassium hydroxide solution, sunflower oil, and n-hexane. There are two possibilities when the samples are soaked in the medium. For the first one, the tiny molecules of medium infiltrated into samples, resulting in a raise of weight. For the second one, some additives in samples were extracted, resulting in a reduction of weight. The two possibilities can take place simultaneously or separately. These are the two opposite processes, and depending on which possibilities prevail, the weight of samples after soaking can raise or reduce compared to the original weight of them. It can be noticed from Figure 2, the weight of samples raised when water and $30 \mathrm{wt} . \%$ acetic solution was used as media and reduced in $10 \mathrm{wt} . \%$ potassium hydroxide solution, sunflower oil, and nhexane. This proved that the tiny molecules of media infiltrated into samples and some additives as DOP, EeRSO, etc., were extracted and entered the media. Specifically, in the water and $30 \mathrm{wt} . \%$ acetic solution, the infiltrative process is more dominant, while in $10 \mathrm{wt} . \%$ potassium hydroxide solution, sunflower oil and $n$-hexane were the extractive process. This experimental data is consistent with the results of the previous study [24].

The hydrophilic property of rubber seed oil is greater than that of DOP, which may be the reason for more the tiny molecules of water or $30 \mathrm{wt} . \%$ acetic solution to penetrate into the 50/10 sample than the $60 / 0$ one, so the weight of $50 / 10$ sample in these media was bigger (see Figure 2(a)). On the contrary, the weight loss of the 50/10 sample in the remaining media was smaller than that of the $60 / 0$ sample, indicating that the EeRSO had a positive effect in reducing the extraction of additives in material into the media. The weight loss of samples was the biggest in $n$-hexane and the smallest in $10 \mathrm{wt} \%$ potassium hydroxide solution (see Figure 2). Since the solubility parameters of PVC (will be shown below) and water near (23.5 MPa [28]) are nearly equal, water molecules will penetrate into the PVC when it is immersed in water; additives such as DOP will be extracted from the PVC and enter the medium where it is hydrolysed. However, since DOP is an ester, it is more easily hydrolysed in potassium hydroxide solution than in acetic acid solution or in water. Therefore, more DOP will be extracted in potassium hydroxide solution medium. This result can lead to the water molecules penetrating into PVC are less than the amount of DOP extracted from the PVC, so the sample weight is reduced. As for water and acetic acid solution, the results are in reverse.

By replacing $10 \mathrm{phr}$ DOP with EeRSO, the module decreased by $8.7 \%$ (see Figure 1), so the shore A hardness (in the air) of PVC with EeRSO was also 6.4\% smaller than that of PVC without EeRSO. The extraction of additives or the penetration of environmental molecules into PVC materials all affect the properties of the material. It may become stiffer or more flexible. The gain or loss of weight is observed in Figure 2(a) showing that there was an infiltration of the tiny environmental molecules into the material and also the extraction of additives from the material into the media. However, the gain or loss weight of the $60 / 0$ sample was only 


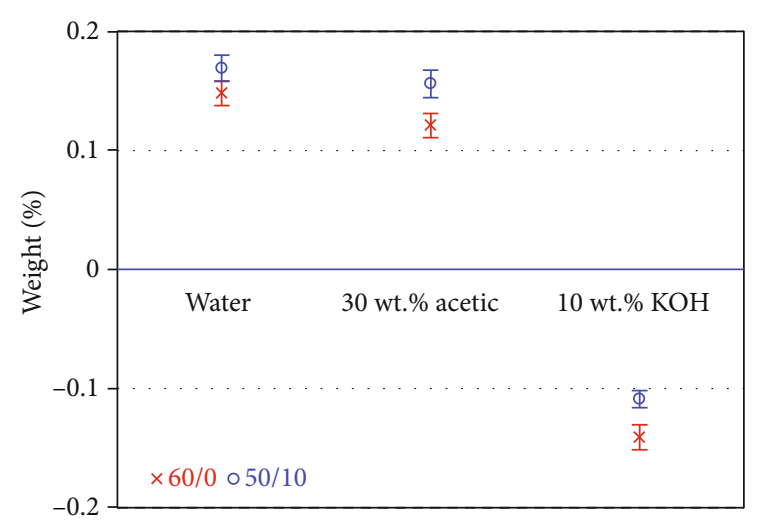

(a)

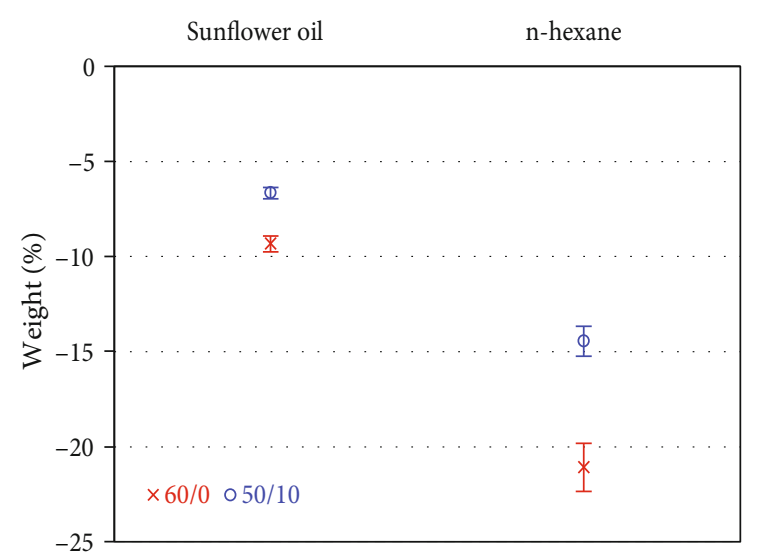

(b)

Figure 2: The effect of EeRSO on the leaching property of PVC in media: (a) water, acid, and base; (b) sunflower oil and n-hexane.

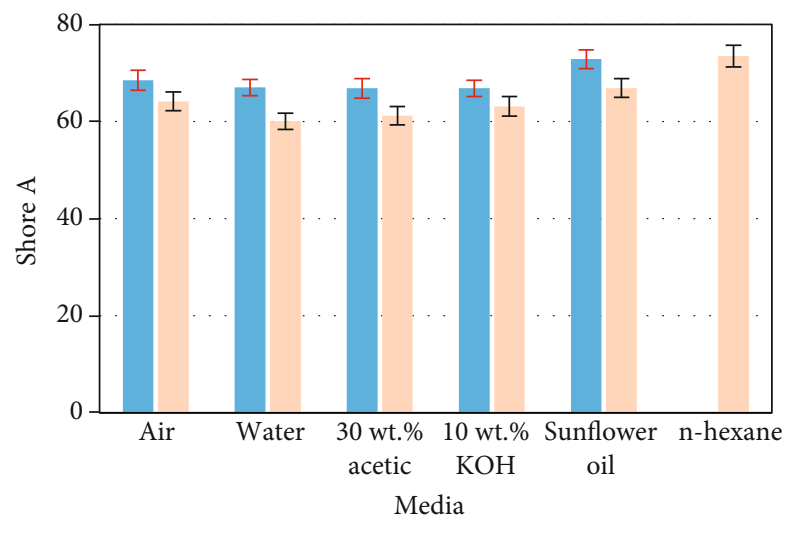

$(60 / 0)$

$(50 / 10)$

FIgUre 3: The shore A of PVC after 72 hours of soaking in media.

about $\pm 0.15 \%$, so the hardness of PVC was almost unchanged after 72 hours of soaking in media as water, $30 \mathrm{wt} . \%$ acetic, and $10 \mathrm{wt} . \%$ potassium hydroxide solution. The result was similar to samples of 50/10 soaked in $10 \mathrm{wt} . \%$ potassium hydroxide solution (see Figure 3). Although the experiment showed that the extraction of the additives from the PVC materials dominated over the phenomenon of tiny molecules of $10 \mathrm{wt} . \%$ potassium hydroxide solution penetrating into the materials, but the hardness of these samples after soaking was still equal to that of nonimmersed samples. This proved that the consequence of the penetration of environmental molecules into the materials overcame the negative effect on hardness due to the extraction of additives. However, in water and $30 \mathrm{wt} . \%$ acetic solution, it was different. After 72 hours of soaking, the gain weight of the 50/10 sample was slightly bigger than that of the 60/0 sample, indicating that the tiny environmental molecules were more absorbed into the samples. The tiny molecules penetrated deep into the material, weaved through the macromolecules chain, making the macromolecular chains more flexible, resulting in less hard materials. Therefore, the shore A hardness of 50/10 samples after 72 hours immersing in these media was slightly lower than that of air medium (see Figure 3).
Unlike in water and the aqueous solution of acid or base, a much larger number of additive molecules was extracted from the PVC materials (see Figure 2(b)) causing a very strong increase in hardness of PVC materials after immersing in organic solvent (n-hexane) and vegetable oil (sunflower oil) (see Figure 3). In this case, the consequence of infiltration of environmental molecules into the materials could not overcome the negative effect on hardness due to the too large additive extraction.

As can be seen from Figure 3, in the air environment, the hardness of the 50/10 sample was slightly lower than that of the 60/0 sample, indicating that the presence of EeRSO had increased the flexibility of the macromolecular chain, thus reducing the hardness of PVC material. The positive role of EeRSO was the most evident when samples immersed in $n$-hexane solvent. The hardness of the 50/10 sample increased from 64.1 to 73.4 , while the $60 / 0$ sample after immersing became extremely stiff, exceeding the scale of Teclock GS 709 machine. The result demonstrated that the partial replacement of DOP plasticizer by EeRSO has improved the extraction properties of PVC materials. This result is similar to the obtained outcome when DOP was partially replaced by ELO in previous publication [24].

After soaking in $\mathrm{n}$-hexane, in addition to additives being extracted from the material, the 60/0 sample was blistered, the sample thickness increased dramatically (see Figure 4(b)), and there were big gaps forming on the cross-section at the blistering place (see Figure 4(c)). This phenomenon did not occur on the 50/10 sample although its hardness after immersion in $\mathrm{n}$-hexane also increased. Due to solubility that is $0.659 \mathrm{~g} / \mathrm{l}$, the PVC will swell in $\mathrm{n}$-hexane [29]. On the other hand, the solubility parameter of PVC, DOP, and EeRSO calculated according to document [30] is $22.57,34.94$, và $19.22 \mathrm{MPa}^{0.5}$, respectively. The solubility parameter of DOP is much larger and of EeRSO is slightly smaller than that of PVC, so the compatibility and miscibility of DOP are worse. This means that the DOP is easier to migrate to the material surface and dissolve in $\mathrm{n}$-hexane. This extraction will form a lot of microholes, and the PVC will be in more contact with the n-hexane, so the PVC will swell strongly. Furthermore, the dehydrochlorination will reduce the molecular weight 


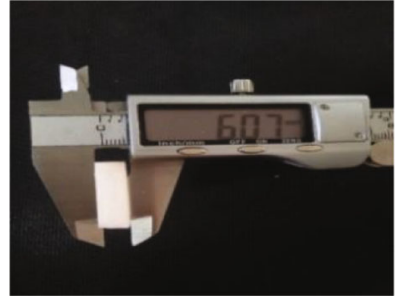

(a)

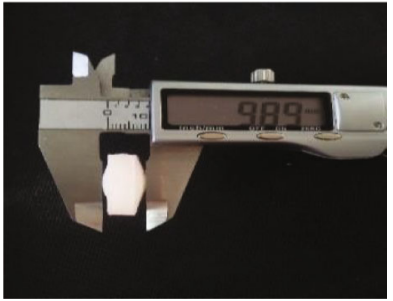

(b)

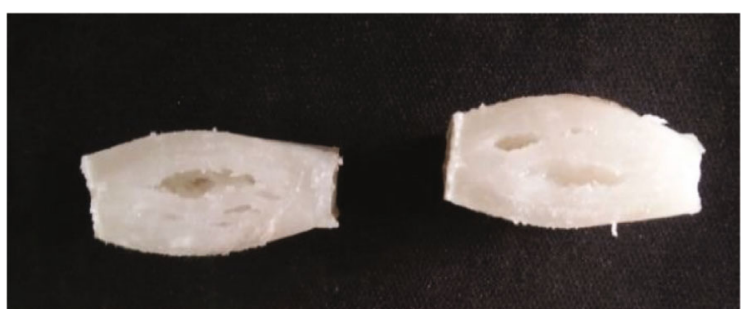

(c)

Figure 4: The picture of 60/0 sample: (a) before, (b) after, and (c) cross-sections after immersion in n-hexane.

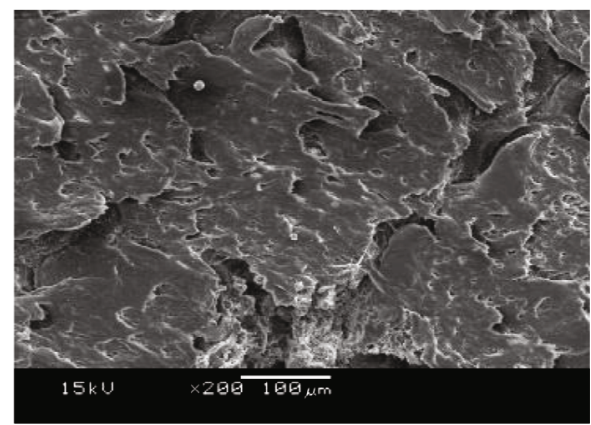

(a)

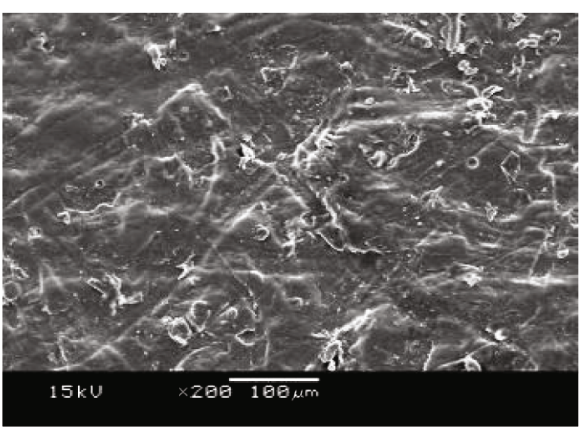

(b)

Figure 5: The SEM of 60/0 (a) and 50/10 (b) samples after immersing in n-hexane.

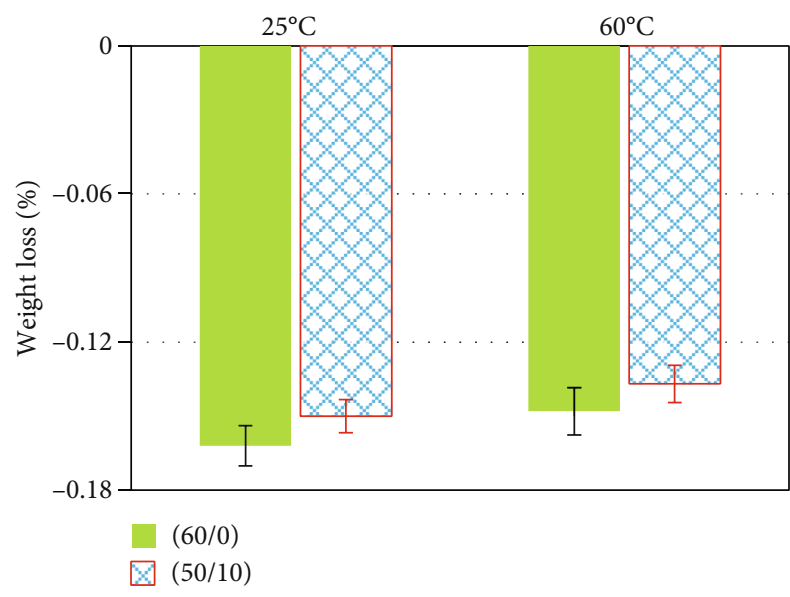

Figure 6: The effect of EeRSO on migration property of PVC material.

of PVC, so the swelling of PVC increased. These are the reasons for the sharp increase in the 60/0 sample thickness. As for the 50/10 sample, the epoxy groups react with the $\mathrm{HCl}$ generated by the dehydrochlorination [10, 25], thus limiting

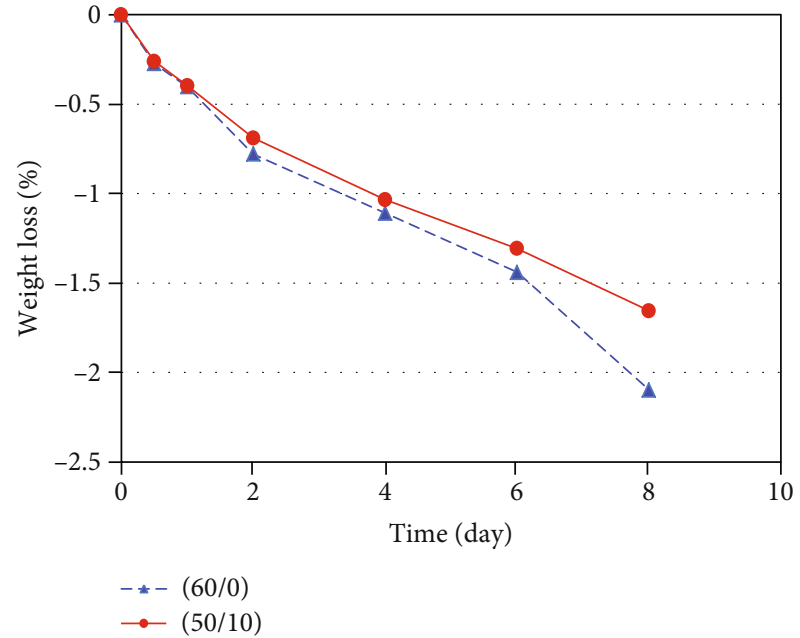

Figure 7: The effect of EeRSO on migration and volatilization of PVC.

the reduction of molecular weight of PVC, resulting in minimizing the swelling of PVC. 


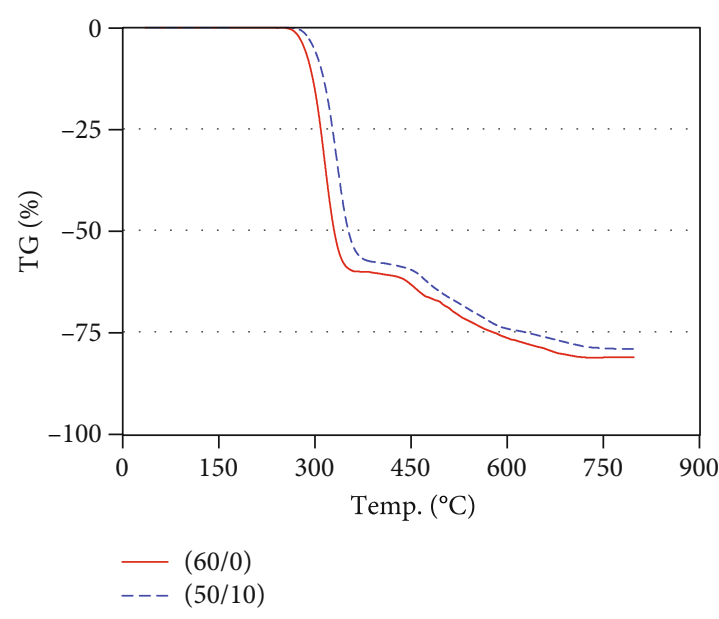

(a)

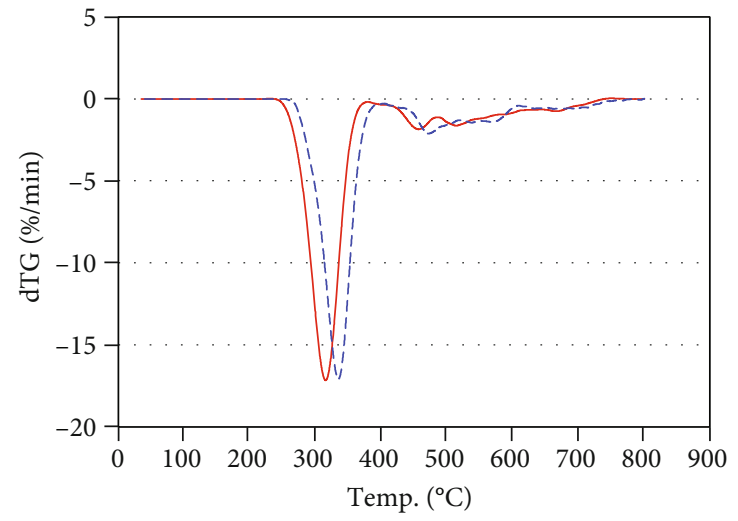

(b)

Figure 8: TG (a) and dTG (b) thermograms of 60/10 and 50/10 samples.

The morphology of the fractured surface of $60 / 0$ and $50 / 10$ samples after 72 hours soaking in $n$-hexane is shown in Figure 5. The extraction of additives from the material left a lot of holes and cracks on the fractured surface of the 60/0 sample (see Figure 5(a)), while they were very difficult to observe on the fractured surface of the 50/10 sample (see Figure 5(b)) at a magnification of 200. This result once again confirmed that the presence of EeRSO had helped to minimize the extraction of additives from the PVC material.

3.3. Migration. It was found that the movement of additives from the inside to the material surface over time will make the PVC material more and more brittle. Epoxidized vegetable oil is known not only as a friendly secondary plasticizer but also has a positive effect in reducing the movement of plasticizers to the surface of the material $[11,24,25]$. As can be seen from Figure 6, the weight of additives moving to the surface after 48 hours at $60^{\circ} \mathrm{C}$ or 2 weeks at $25^{\circ} \mathrm{C}$ of the 50/10 sample was smaller than that of the 60/10 sample. It was confirmed that EeRSO is not only effective in plasticizing PVC (see Figure 1) but also had a positive influence on reducing the migration of additives to the surface of the material. Moreover, the amount of the additives migrated for a short time at high temperatures was less than at low temperatures but for a long time (see Figure 6).

3.4. Migration and Volatilization in Hot Air. In this test, the additive that moved to the surface of the material and was volatilized in hot air instead of getting absorbed in the filter paper as the migration test. As can be seen from Figure 7, the weight loss of samples increased over time. The weight loss of two samples was the same in the first day. After that, the weight loss of the 50/10 sample was smaller than that of the $60 / 0$ sample. The longer the test period was, the greater the difference in the weight loss was. The weight loss of $50 / 10$ and 60/0 samples after 8 test days was 1.65 and $2.09 \%$, respectively. That means the movement and volatilization of additives in PVC material in hot air would be reduced with the presence of EeRSO.
TABLE 2: The effect of EeRSO on the thermal stability of PVC material.

\begin{tabular}{lccccc}
\hline \multirow{2}{*}{ Sample } & \multicolumn{3}{c}{$1^{\text {st }}$ stage } & \multicolumn{2}{c}{$2^{\text {nd }}$ stage } \\
& $T_{\text {onset }}$ & $T_{1 \max }$ & $\%$ weight loss & $T_{2 \max }$ & $\%$ weight loss \\
\hline $60 / 0$ & 232.06 & 315.03 & 60.89 & 455.65 & 20.54 \\
$50 / 10$ & 251.24 & 334.58 & 58.13 & 472.01 & 21.17 \\
\hline
\end{tabular}

3.5. Thermal Property. The $50 / 10$ and $60 / 0$ samples were subjected to thermogravimetric analysis and the data used to estimate the impact of EeRSO on the degradation of PVC material. Figure 8 shows the TG and dTG curves of PVC materials with or without EeRSO were heated in the air at the rate of $10^{\circ} \mathrm{C} / \mathrm{min}$ from room temperature to $800^{\circ} \mathrm{C}$. The obtained results for degradation processes such as an inset temperature $\left(T_{\text {onset }}\right)$, which is the initial weight loss temperature, a maximum degradation temperature $\left(T_{\max }\right)$, which is the temperature of a maximum rate of degradation and weight loss in percent of each stage are shown in Table 2.

Previous workers showed that the degradation of PVC material was a two-stage process. The first stage relates to dehydrochlorination $[11,31,32]$ and the thermal decomposition of plasticizers [33]. The second stage corresponds to the formation of hydrocarbon as aromatic compounds by the cyclization of conjugated polyene and to the carbonization of structures formed during the first degradation stage [31-33]. In which, the degradation in the first stage took place very strongly and the sample weight also decreased very quickly due to the release of $\mathrm{HCl}$.

The decomposition curve behaviour of 50/10 sample with the presence of EeRSO was largely similar to that of the 60/0 sample and took place in two stages (see Figure 8(a)), which could be in evidence from the dTG curves in Figure $8(\mathrm{~b})$. The first stage took place up to about $409^{\circ} \mathrm{C}$. The $60 / 0$ sample began to decompose at $232^{\circ} \mathrm{C}$, and it was $20^{\circ} \mathrm{C}$ smaller than the $T_{\text {onset }}$ of the 50/10 sample. When $\mathrm{PVC}$ undergoes heat, $\mathrm{HCl}$ is produced by the dehydrochlorination (1) [10,25]. This newly generated $\mathrm{HCl}$ promotes the dehydrochlorination even more. However, in the presence 
of epoxidized oils such as EeRSO, the newly generated $\mathrm{HCl}$ will react with the epoxy groups of EeRSO (2), thus inhibiting the dehydrochlorination. Thus, the $T_{\text {onset }}$ and $T_{1 \max }$ of the $50 / 10$ sample were higher, while the weight loss of the $50 / 10$ sample in the first stage was lower than that of the 60/0 sample (see Table 2).

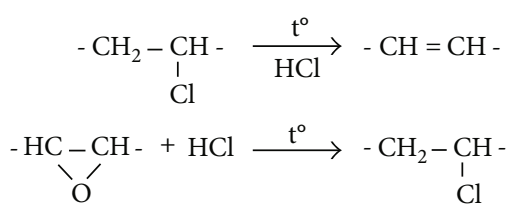

Like the first stage, the presence of EeRSO also increased $T_{2 \max }$ of the second one (see Table 2). Overall, the presence of EeRSO had a positive effect on the thermal stability of PVC material. If comparing the thermal parameters on the TG and dTG curves of the 50/10 sample (see Figure 8) with that of the PVC using $10 \mathrm{phr}$ epoxidized linseed oil (ELO) in published work [24], the thermal stability effect of EeRSO was superior to that of ELO. Therefore, EeRSO, which is a mixture of epoxidized triglycerides and epoxidized methyl esters of free fatty acids can improve the thermal stability of PVC better than ELO with only epoxidized triglycerides.

\section{Conclusions}

The presence of 10 phr EeRSO with components including epoxidized triglycerides and epoxidized methyl esters of free fatty acids has shown an improvement in PVC's flexibility due to an increase in $7 \%$ tensile strength and $11.4 \%$ elongation at break. The leaching test results showed that the presence of 10 phr EeRSO as a secondary plasticizer improved remarkably the leaching characteristic of plasticizers in PVC materials. The morphology of fractured surface the specimens after immersing in $n$-hexane was clear evidence of positive effects of EeRSO to leaching properties. The hardness of PVC material was also significantly improved especially in the $\mathrm{n}$-hexane environment when $10 \mathrm{phr}$ DOP was replaced by EeRSO. The migration characteristic of PVC material with 10 phr EeRSO has been improved strongly at 25 and $60^{\circ} \mathrm{C}$. The positive effect of substituting $10 \mathrm{phr}$ DOP by EeRSO was also represented through thermal properties and movement and volatilization in hot air. Thermogravimetric analysis showed the significant improvement in thermal stability when 10 phr DOP was replaced by EeRSO in PVC formulation. Thus, the presence of EeRSO not only has the effect of plasticizing PVC, reducing the extraction, migration, and volatilization of the additives but also improves the thermal stability for PVC. The overall results showed that epoxidized rubber seed oil, which is a mixture of epoxidized triglycerides and epoxidized methyl esters of free fatty acids, could be used as a secondary plasticizer/thermal stabilizer in PVC formulation. The results of this work have shown that we can save because all the ingredients in high acid value rubber seed oil can be utilized to convert to epoxy form for use as a secondary plasticizer/thermal stabilizer in PVC processing.

\section{Data Availability}

The data used to support the findings of this study are included within the article.

\section{Conflicts of Interest}

The authors declare that there is no conflict of interest regarding the publication of this paper.

\section{Acknowledgments}

This work was supported by the National Key Laboratory for Polymer \& Composite Materials, Hanoi University of Science and Technology, and the authors also thank Mr. Vu Quang Truong for his assistant.

\section{References}

[1] B. L. Shah and V. V. Shertukde, "Effect of plasticizers on mechanical, electrical, permanence, and thermal properties of poly(vinyl chloride)," Journal of Applied Polymer Science, vol. 90, no. 12, pp. 3278-3284, 2003.

[2] H. Hosney, B. Nadiem, I. Ashour, I. Mustafa, and A. El-Shibiny, "Epoxidized vegetable oil and bio-based materials as PVC plasticizer," Journal of Applied Polymer Science, vol. 135, no. 20, article 46270, 2018.

[3] F. E. Okieimen, T. O. Egbuchunam, and D. Balköse, "Studies in the utilization of biobased additives as thermal stabilizer for plasticized PVC," Advanced Materials Research, vol. 62-64, pp. 335-344, 2009.

[4] N. Gil, M. Saska, and I. Negulescu, "Evaluation of the effects of biobased plasticizers on the thermal and mechanical properties of poly(vinyl chloride)," Journal of Applied Polymer Science, vol. 102, no. 2, pp. 1366-1373, 2006.

[5] H. B. Pyeon, J. E. Park, and D. H. Suh, "Non-phthalate plasticizer from camphor for flexible PVC with a wide range of available temperature," Polymer Testing, vol. 63, pp. 375-381, 2017.

[6] T. Liu, P. Jiang, H. Liu et al., "Performance testing of a green plasticizer based on lactic acid for PVC," Polymer Testing, vol. 61, pp. 205-213, 2017.

[7] F. E. Okieimen and J. E. Ebhoaye, "Thermal dehydrochlorination of PVC in the presence of rubber seed oil," Die Angewandle Makoromolekulare Chemie, vol. 206, no. 1, pp. 11-20, 1993.

[8] F. E. Okieimen, J. E. Ebhoaye, and E. E. Justus, "Studies in the thermal degradation of poly(vinyl chloride)," Journal of Applied Polymer Science, vol. 48, no. 10, pp. 1853-1858, 1993.

[9] R. Joseph, K. N. Madhusoodhanan, R. Alex, S. Varghese, K. E. George, and B. Kuriakose, "Studies on epoxidised rubber seed oil as secondary plasticiser/stabiliser for polyvinyl chloride," Plastics Rubber and Composites, vol. 33, no. 5, pp. 217-222, 2004.

[10] T. O. Egbuchunam, D. Balköse, and F. E. Okieimen, "Effect of zinc soaps of rubber seed oil (RSO) and/or epoxidised rubber seed oil (ERSO) on the thermal stability of PVC plastigels," Polymer Degradation and Stability, vol. 92, no. 8, pp. 15721582, 2007.

[11] D. Balköse, T. O. Egbuchunam, and F. E. Okieimen, "Formulation and properties' evaluation of $\mathrm{PVC} /$ (dioctyl phthalate)/(epoxidized rubber seed oil) plastigels," Journal of 
Vinyl and Additive Technology, vol. 14, no. 2, pp. 65-72, 2008.

[12] J. Frados, Plastics Engineering Handbook, of the Society of the Plastics Industry, Van Nostrand Reinhold, New York, USA, 4th ed. edition, 1976.

[13] L. Krauskopf, "Monomeric plasticizers," in Handbook of Polyvinyl Chloride Formulating, E. J. Wickson, Ed., pp. 216-219, Wiley, New York, USA, 1993.

[14] J. D. Meeker, S. Sathyanarayana, and S. H. Swan, "Phthalates and other additives in plastics: human exposure and associated health outcomes," Philosophical Transactions of the Royal Society B: Biological Sciences, vol. 364, no. 1526, pp. 2097-2113, 2009.

[15] M. de Fátima Poças and T. Hogg, "Exposure assessment of chemicals from packaging materials in foods: a review," Trends in Food Science \& Technology, vol. 18, no. 4, pp. 219-230, 2007.

[16] M. G. A. Vieira, M. A. da Silva, L. O. dos Santos, and M. M. Beppu, "Natural-based plasticizers and biopolymer films: a review," European Polymer Journal, vol. 47, no. 3, pp. 254263, 2011.

[17] L. Coltro, J. B. Pitta, and E. Madaleno, "Performance evaluation of new plasticizers for stretch PVC films," Polymer Testing, vol. 32, no. 2, pp. 272-278, 2013.

[18] C. Bueno-Ferrer, M. C. Garrigós, and A. Jiménez, "Characterization and thermal stability of poly(vinyl chloride) plasticized with epoxidized soybean oil for food packaging," Polymer Degradation and Stability, vol. 95, no. 11, pp. 2207-2212, 2010.

[19] P. Karmalm, T. Hjertberg, A. Jansson, and R. Dahl, “Thermal stability of poly(vinyl chloride) with epoxidised soybean oil as primary plasticizer," Polymer Degradation and Stability, vol. 94, no. 12, pp. 2275-2281, 2009.

[20] P. Karmalm, T. Hjertberg, A. Jansson, R. Dahl, and K. Ankner, "Network formation by epoxidised soybean oil in plastisol poly(vinyl chloride)," Polymer Degradation and Stability, vol. 94, no. 11, pp. 1986-1990, 2009.

[21] O. Fenollar, D. Garcia-Sanoguera, L. Sanchez-Nacher, J. López, and R. Balart, "Effect of the epoxidized linseed oil concentration as natural plasticizer in vinyl plastisols," Journal of Materials Science, vol. 45, no. 16, pp. 4406-4413, 2010.

[22] O. Fenollar, D. Garcia-Sanoguera, L. Sánchez-Nácher, J. López, and R. Balart, "Characterization of the curing process of vinyl plastisols with epoxidized linseed oil as a natural-based plasticizer," Journal of Applied Polymer Science, vol. 124, no. 3, pp. 2550-2557, 2012.

[23] M. P. Arrieta, M. D. Samper, M. Jiménez-López, M. Aldas, and J. López, "Combined effect of linseed oil and gum rosin as natural additives for PVC," Industrial Crops and Products, vol. 99, pp. 196-204, 2017.

[24] N. T. Thuy and V. M. Duc, "The epoxidized linseed oil as a secondary plasticizer in PVC processing," Vietnam Journal of Chemistry, vol. 58, no. 4, pp. 533-538, 2020.

[25] M. T. Benaniba and V. Massardier-Nageotte, "Evaluation effects of biobased plasticizer on the thermal mechanical, dynamical mechanical properties, and permanence of plasticized PVC," Journal of Applied Polymer Science, vol. 118, no. 6, pp. 3499-3508, 2010.

[26] N. Saetung, S. Somjit, P. Thongkapsri, T. Tulyapitak, and A. Saetung, "Modified rubber seed oil based polyurethane foams," Journal of Polymer Research, vol. 23, no. 3, p. 58, 2016.

[27] J. Chen, X. Li, Y. Wang et al., "Epoxidized dimeric acid methyl ester derived from rubber seed oil and its application as sec- ondary plasticizer," Journal of Applied Polymer Science, vol. 133, no. 34, article 43668, 2016.

[28] C. M. Hansen, The Three Dimensional Solubility Parameter and Solvent Diffusion Coefficient, Their Importance in Surface Coating Formulation, Copenhagen Danish Technical Press, 1967.

[29] G. Grause, S. Hirahashi, H. Toyoda, T. Kameda, and T. Yoshioka, "Solubility parameters for determining optimal solvents for separating PVC from PVC-coated PET fibers," Journal of Material Cycles and Waste Management, vol. 19, no. 2, pp. 612-622, 2017.

[30] D. W. Van Krevelen and K. Te Nijenhuis, "Properties of Polymers-Their Correlation with Chemical Structure" Their Numerical Estimation and Prediction from Additive Group Contributions, 4th., Elsevier B. V, 2009.

[31] J. Jimenez, A. I. Lopez, A. Iannoni, and J. M. Kenny, "Formulation and mechanical characterization of PVC plastisols based on low-toxicity additives," Journal of Applied Polymer Science, vol. 81, no. 8, pp. 1881-1890, 2001.

[32] C. E. Sogbaike, F. E. Okieimen, and O. C. Eromosele, "Thermo-oxidative degradation of poly(vinyl chloride) in the presence of derivatives of Khaya seed oil," Polymer Degradation and Stability, vol. 88, no. 2, pp. 175-181, 2005.

[33] D. A. Burg and R. Kleiman, "Preparation of meadowfoam dimer acids and dimer esters, and their use as lubricants," Journal of the American Oil Chemists' Society, vol. 68, no. 8, pp. 600-603, 1991. 